\title{
Study on the Degradation Effect of Plant-Derived Active Ingredients on Organophosphorus Pesticides
}

\section{Jun Sun ${ }^{*}$ Tongxin Zhang}

Shandong Rengjian Biotechnology Co., Ltd., Jinan, Shandong, 250200, China

\begin{tabular}{l}
\hline ARTICLE INFO \\
\hline Article history \\
Received: 19 March 2020 \\
Revised: 26 March 2020 \\
Accepted: 24 April 2020 \\
Published Online: 30 April 2020 \\
\hline
\end{tabular}

\section{Keywords:}

Organophosphorus pesticides

Plant-Derived active ingredients

Pesticide degradation

Chinese medicine agriculture

Soil remediation

\begin{abstract}
In order to explore new ways and methods for the degradation of organophosphorus pesticides, the degradation effects of plant-derived active ingredients on three organophosphorus pesticides were studied. Rhubarb, Pittosporum bark, Hibiscus bark, and Chinese gall in 9:4:3:2 parts were mixed by mass, ground and soaked in water, and the concentration changes of organic phosphorus pesticide before and after the test were compared through GC-MS quantitative detection method and rapid pesticide residue measurement method to clarify its degradation effect on organophosphorus pesticides. The results showed that the degradation rates of chlorpyrifos and parathion were $93.2 \%$ and $92.9 \%$ respectively in the extract within 2 minutes; the degradation rate of dichlorvos was $66.67 \%$ in the extract within 17 hours, and the degradation rate of chlorpyrifos was $48.69 \%$ within 11 hours. This study shows that the extracts of rhubarb, sea tongs bark, hibiscus bark, and gallnut have significant degradation effects on chlorpyrifos, parathion, dichlorvos and other organophosphorus pesticides.
\end{abstract}

pecially in the field of pesticides, accounting for more than $70 \%$ of the total pesticides ${ }^{[6]}$. The utilization rate of pesticides applied in farmland is $38.8 \%{ }^{[7]}$. Most of the pesticides diffuse into the environment, enter the human body through the water vapor cycle and the food chain, endangering health, and because a large amount of pesticides remain in the soil, they become agricultural non-point source pollution. One of the important factors ${ }^{[8-9]}$

Pesticide degradation can be divided into physical methods, chemical methods and biological methods. However, due to factors such as application conditions, use costs, and technological maturity, large-scale promotion and application have not been carried out ${ }^{[8,10]}$,

*Corresponding Author:

Jun Sun,

Shandong Rengjian Biotechnology Co., Ltd., Jinan, Shandong, 250200, China;

E-mail: sj@nassg.org 
especially in agricultural production. There is no precedent. At present, biological methods are considered to be an ideal method to reduce pesticide residues due to their significant effect of degrading pesticide residues and low environmental impact. In particular, the screening and application technology research and development of pesticide degrading bacteria have achieved significant research results ${ }^{[8,10-12]}$, but these technologies are still in the laboratory development stage.

Based on the principle of enzymatic degradation of pesticides, this research has developed a unique approach, based on the theory of traditional Chinese medicine, prescriptions and years of application practice, to screen and develop plant-derived active ingredients that have rapid and efficient degradation effects on organophosphorus pesticides. After indoor testing and field verification, The effect is remarkable, realizing the cross-border integration of modern agriculture and traditional Chinese medicine, and providing a good technical path and basic data support for exploring and applying Chinese medicine concepts to promote the harmonious development of "production, life, and ecology". It has important scientific research and application value.

\section{Materials and Methods}

\subsection{Preparation of Plant-Derived Active Ingredients}

(1) Mix and crush the raw materials of traditional Chinese medicine such as $180 \mathrm{~g}$ of rhubarb, $80 \mathrm{~g}$ of sea tongs bark, $60 \mathrm{~g}$ of hibiscus bark, $40 \mathrm{~g}$ of gallnut, and sieving through 200 mesh.

(2) Put the powder in a stainless steel container, add $2000 \mathrm{~g}$ of water, and heat to $100^{\circ} \mathrm{C}$ for $30 \mathrm{~min}$.

(3) Under the condition of full contact with air, cool down naturally (not lower than $5^{\circ} \mathrm{C}$, and let stand for $48 \mathrm{~h}$.

(4) Filter out the drug solution and dilute it with 5,000 $\mathrm{g}$ of clean water (the water temperature is room temperature).

\subsection{Detection of Degradation Effect}

\subsubsection{Gas Chromatography-Mass Spectrometry (GC-MS Method)}

March 10, 2018, in the laboratory of the Institute of Agricultural Quality Standards and Testing Technology, Shandong Academy of Agricultural Sciences, using Agilent $7890 \mathrm{~A}$ gas chromatography-mass spectrometry, the analytical method quantitatively detects the rapid degradation effect of plant-derived components on organophosphorus pesticides (chlorpyrifos, parathion) ${ }^{[13-15]}$.

(1) Prepare a $1 \mu \mathrm{g} / \mathrm{mL}$ mixed standard sample of chlorpyrifos and parathion.

(2) Take $1 \mathrm{~mL}$ of the standard sample and place it in a $20 \mathrm{~mL}$ pointed glass test tube, then add $1 \mathrm{~mL}$ of plant-derived active ingredient solution, and vortex for $1 \mathrm{~min}$.

(3) Add $5 \mathrm{~mL}$ acetonitrile and $1 \mathrm{~g} \mathrm{NaCl}$, and vortex for $1 \mathrm{~min}$

(4) Centrifuge at 3,000 r/min for $2 \mathrm{~min}$, take it out and place it in a test tube rack.

(5) Take $1 \mathrm{~mL}$ of the supernatant solution after centrifugation in a glass test tube, blow with nitrogen in a $40^{\circ} \mathrm{C}$ water bath (until it blows dry), and add $2 \mathrm{~mL}$ of acetone to make the volume constant.

(6) On-machine (Agilent 7890A) detection, the injection volume is $2 \mu \mathrm{L}$. Set 3 repeats.

\subsubsection{Pesticide Residue Rapid Detection Method}

With leek and chrysanthemum chrysanthemum as the test objects, the NC-800 6-channel intelligent pesticide residue rapid detector is used to detect the degradation effect of plant-derived components on organophosphorus pesticides (dichlorvos, chlorpyrifos).

(1) Leek-Dichlorvos

Test conditions: On May 6, 2018, the experiment was carried out at the leek base in Caiyuan Village, Zhanglu Town, Xin County, Liaocheng City, Shandong Province. The base was planted with leek for 2 years, covering an area of $0.33 \mathrm{hm}^{2}$, with a variety of Hanzhong winter leek and a plant height of $15-20 \mathrm{~cm}$., The border is east-west, $25 \mathrm{~m}$ long, $2 \mathrm{~m}$ wide, and leeks are planted horizontally from north to south. The experiment was carried out on May 6, with real-time temperature $22-25^{\circ} \mathrm{C}$, wind force $2-3 ; 16-17$ hours later, sampling and testing.

Experimental procedure:

(1) From $15: 00$ to $16: 00$ on May 6 th, select a leek plot of $20 \mathrm{~m}^{2}$, and spray the leek plants and the ground between rows with $775 \mathrm{mg} / \mathrm{L}$ dichlorvos 2,000 mL evenly;

(2) Randomly select $10 \mathrm{~m}^{2}$ of them for test treatment, immediately dilute with plant-derived active ingredients and room temperature water by 4 times, take $400 \mathrm{~mL}$ and spray evenly on the leek plants and the ground between rows; the other $10 \mathrm{~m}^{2}$ is used as control I;

(3) The same planting conditions of adjacent plots, without spraying dichlorvos and plant-derived active ingredients of leek in the leek border as control II;

(4) From 9 am to 10 am on May 7th, use the 5-point mixed sampling method to collect $1 \mathrm{~kg}$ of leek samples in the experimental treatment area, control I area, and control II area, and put them into plastic sealed bags; at 11 o'clock, use the rapid test method Detection of dichlorvos residues 
in leek samples. Let 2 repeats.

(2) Garland chrysanthemum-Chlorpyrifos

Test conditions: On May 9, 2018, the experiment was carried out at the Chrysanthemum chrysanthemum export base in Shiziyuan Town, Xin County, Liaocheng City, Shandong Province. The base planted Chrysanthemum chrysanthemum $2.67 \mathrm{hm}^{2}$, the growth period was 45 days, the plant height was $30-50 \mathrm{~cm}$, and the border was eastwest., $80 \mathrm{~m}$ long and $5 \mathrm{~m}$ wide, Chrysanthemum vulgare was planted vertically from east to west. It was harvested on May 10th as planned. Due to accidental spraying during flight, chlorpyrifos exceeded the standard by 16 times. Emergency treatment should be taken upon request. The test was carried out at night on May 9. The real-time temperature is $16-18^{\circ} \mathrm{C}$, without wind; it will be detected after 10-11 hours.

(3) Experimental Procedure

(1) Set up 3 communities in Qitou and Qizhong, each of which is about $20 \mathrm{~m}^{2}$;

(2) At 22:00 on the evening of May 9th, dilute 4 times with plant-derived active ingredients and room temperature water, and spray 1,000 mL evenly on Chrysanthemum chrysanthemum plants in the test plot;

(3) Take the adjacent section on the west side of each plot as a control;

(4) At 8:00 on May 10, use the 5-point mixed sampling method to collect $500 \mathrm{~g}$ of Chrysanthemum chrysanthemum (middle and upper part) samples in the experimental treatment area and the control area, and put them into plastic bags; at 9:00, use the rapid measurement method Detection of chlorpyrifos residues in Chrysanthemum chrysanthemum samples.

\section{Results and Analysis}

\subsection{The Degradation Effect of Plant-Derived Ac- tive Ingredients on Chlorpyrifos and Parathion}

T-test analysis was performed by SPSS, and the results are shown in Table 1. Taking the test concentration of the standard sample as the initial concentration (ck), after mixing the chlorpyrifos and parathion standard sample solution with the plant-derived active ingredients for 2 minutes, the degradation rate of chlorpyrifos is $93.6 \%$, the degradation rate of parathion is $92.9 \%$, and the degradation rate of chlorpyrifos and parathion is $92.9 \%$. Phosphorus concentration decreased significantly $(\mathrm{P}<0.01)$, and the degradation effect was significant; the degradation effects of chlorpyrifos and parathion were equivalent, and the difference did not reach a significant level $(\mathrm{t}=2.347$, $\mathrm{P}=0.079$ )
Table 1. Degradation effects of plant-derived active ingredients on chlorpyrifos and parathion (indoor)

\begin{tabular}{|c|c|c|c|c|}
\hline species & $\begin{array}{c}\text { Test } \\
\text { repeat }\end{array}$ & $\begin{array}{c}\text { Concentra- } \\
\text { tion } /(\mu \mathrm{g} / \mathrm{mL})\end{array}$ & $\begin{array}{l}\text { Mean } /(\mu \mathrm{g} / \mathrm{mL}) \pm- \\
\text { standard deviation }\end{array}$ & $\begin{array}{l}\text { Degrada- } \\
\text { tion rate } \%\end{array}$ \\
\hline \multirow{3}{*}{ samples } & 1 & 0.9635 & \multirow{3}{*}{$0.9653 \pm 0.0047 \mathrm{~A}$} & \\
\hline & 2 & 0.9706 & & \\
\hline & 3 & 0.9618 & & \\
\hline \multirow{3}{*}{$\begin{array}{l}\text { Chlorpy- } \\
\text { rifos }\end{array}$} & 1 & 0.0983 & \multirow{3}{*}{$0.0962 \pm 0.0032 \mathrm{~B}$} & \multirow{3}{*}{93.6} \\
\hline & 2 & 0.0978 & & \\
\hline & 3 & 0.0925 & & \\
\hline \multirow{3}{*}{$\begin{array}{l}\text { Parathi- } \\
\text { on }\end{array}$} & 1 & 0.1039 & \multirow{3}{*}{$0.1031 \pm 0.0039 \mathrm{~B}$} & \multirow{3}{*}{92.9} \\
\hline & 2 & 0.1065 & & \\
\hline & 3 & 0.0988 & & \\
\hline
\end{tabular}

\subsection{The Field Degradation Effect of Plant-Derived Active Ingredients on Dichlorvos and Chlorpyrifos}

It can be seen from Table 2 that the control II leek sample is $1.01 \%$, the control leek sample is $19.00 \%$, and the experimental treatment leek sample is $6.11 \%$. The results show that the plant-derived active ingredients have a significant effect on dichlorvos under natural conditions in the field, within 17 hours the degradation rate reached $66.67 \%$.

Table 2. Degradation effect of plant-derived active ingredients on dichlorvos (field)

\begin{tabular}{|c|c|c|c|c|}
\hline species & Test repeat & Inhibition rate/\% & Mean/\% & Degradation rate/\% \\
\hline \multirow{2}{*}{ CKI } & 1 & 0.48 & \multirow{2}{*}{1.01} & \\
\cline { 2 - 3 } & 2 & 1.54 & & \\
\hline \multirow{2}{*}{ CKII } & 1 & 20.41 & \multirow{2}{*}{19.00} & \\
\cline { 2 - 3 } & 2 & 17.59 & & \\
\hline \multirow{2}{*}{$\begin{array}{c}\text { Test treat- } \\
\text { ment }\end{array}$} & 1 & 11.89 & \multirow{2}{*}{6.11} & 66.67 \\
\cline { 2 - 3 } & 2 & 0.32 & & \\
\hline
\end{tabular}

It can be seen from Table 3 that after emergency treatment, the concentration of chlorpyrifos on Chrysanthemum chrysanthemum was significantly reduced. After 10 to 11 hours, the degradation rate exceeded $48.7 \%$.

Because $100 \%$ of the pesticide residue concentration has exceeded the instrument range, it is impossible to obtain a relatively accurate value. Although it is impossible to perform an accurate significance test and analysis, it is inferred from the data distribution that there should be a significant difference between the two.

DOI: http://dx.doi.org/10.36956/rwae.v1i1.162 
Table 3. Degradation effect of plant-derived active ingredients on chlorpyrifos (field)

\begin{tabular}{|c|c|c|c|c|}
\hline species & Test repeat & Inhibition rate/\% & Mean/\% & Degradation rate/\% \\
\hline \multirow{2}{*}{$\begin{array}{c}\text { Test } \\
\text { treat- } \\
\text { ment }\end{array}$} & 1 & 52.16 & & \\
\cline { 2 - 3 } & 2 & 46.41 & \multirow{2}{*}{46.83} & 48.69 \\
\hline \multirow{3}{*}{ CK } & 3 & 41.92 & & \\
\cline { 2 - 3 } & 2 & 73.77 & \multirow{2}{*}{91.26} & \\
\cline { 2 - 3 } & 3 & 100 & & \\
\hline
\end{tabular}

\section{Conclusion}

This study shows that the extract obtained from rhubarb, sea tongs bark, hibiscus bark, and Chinese gall according to the fixed mass ratio has a significant degradation effect on chlorpyrifos, parathion, dichlorvos and other organophosphorus pesticides, and the degradation rate is extremely fast. Especially when organophosphorus pesticides are fully mixed, the degradation efficiency exceeds $90 \%$ in 2 minutes.

In view of the rapid and significant degradation effect of this plant-derived component on organophosphorus pesticides, it has broad application prospects in farmland soil remediation, elimination of pesticide residues in fresh fruits and vegetables, production of organophosphorus toxic compounds and emergency treatment of leakage, clinical poisoning rescue, etc.

\section{Discussion}

Organophosphorus pesticides are widely used. With the increase of pest resistance, the amount and frequency of application continue to increase, which has a greater impact on the environment and poses an acute risk of poisoning to the human body ${ }^{[16]}$. Pesticide biodegradation technology is a hotspot of global technology research in the field in recent years ${ }^{[17-18]}$. Obligate and broad-spectrum microbial species with obvious enzymatic degradation of organophosphorus have also been screened from soil and water, including bacteria, Fungi, actinomycetes and algae. Among them, the research on bacteria is the most in-depth ${ }^{[17]}$, such as the more representative Pseudomonas in bacteria, which has good degradation of malathion, dichlorvos, phorate, and methyl parathion effect. Liu Yuhuan et al. ${ }^{[19]}$ conducted a detailed study on methamidophos-degrading fungi, Wang Baihui et al. reviewed the mechanism of soil enzyme degradation of pesticides by microorganisms ${ }^{[20]}$, Zhao Renbang et al. ${ }^{[21]}$ degraded methylamine by Penicillium oxalicum ZHJ6 The application method of phosphorus was studied. Biazyme is a fermentation prod- uct produced by microorganisms selected from the soil. As a result of the "863 Program" project, it has been exclusively produced by Beijing Sengenbia Biotechnology Co., Ltd. since 2012. It is used for civilian purposes. It is against dichlorvos, chlorpyrifos, and Mala. The average degradation effect of thion, fenthion, and methyl parathion reaches $70 \%$, and a number of patented technologies such as "methyl parathion hydrolase mutant with improved enzymatic performance and its application" have been developed $^{[22]}$.

Up to now, there are few research literatures on the application of pure plant-derived ingredients to degrade organophosphorus pesticides and other types of pesticides. As a brand-new concept in the development of modern agriculture, TCM agriculture is of great significance to promoting the sustainable development of agriculture ${ }^{[23]}$. In traditional Chinese medicine, there are many formulas and cases of applying Chinese medicine to detoxify. Edible proteases are extracted from pineapple, papaya and other fruits to develop detergents that can degrade vegetable pesticide residues, such as methamidophos, omethoate, dichlorvos, and chlorpyrifos. The degradation rate of other pesticides reaches more than $90 \%{ }^{[24]}$; different concentrations of tea tree oil and water-soluble tea tree oil are used to soak and clean the cowpea sprayed with pesticides. The results show that $0.8 \%$ of water-soluble tea oil has the best effect in removing pesticide residues. Excellent, the pesticide removal rates for triazophos, malathion, and chlorpyrifos are $82.79 \%, 94.54 \%$, and $84.58 \%$, respectively ${ }^{[25]}$.

Although many kinds of microorganisms and plant extracts that degrade pesticides have been screened out, it can be seen from the existing literature that these research results have not yet been applied on a large scale. As the most stable and effective organophosphorus pesticide degradation enzyme at present, Biazyme is affected by factors such as raw materials, production methods, yield and cost, and its application range is limited. The plant-derived ingredients in the traditional Chinese medicine prescriptions used in this study have a wide range of sources and have equivalent functional alternatives, which can be mass-produced at low cost. After 11 years of field application, the effect is significant and stable. For the first time in this study, the principles and techniques of detoxification of traditional Chinese medicine were applied to the degradation of chemical pesticides. The prescriptions of traditional Chinese medicines that can efficiently degrade organophosphorus pesticides were screened out, and more systematic production processes, products and supporting application technologies were developed, which can be applied to farmland. Degradation of pesticide residues in 
contaminated soil, degradation of pesticide residues on agricultural products, water environment disinfection, and emergency treatment of organophosphorus pesticides (factories) leakage. The field application initially shows that this plant-derived ingredient has a certain degradation effect on carbamates, sulfonylurea pesticides and herbicides, and scientific experiments are needed to prove it; at the same time, the mechanism of action of this ingredient needs further research.

\section{References}

[1] Lin Zhengping. Current status and treatment measures of non-point source pollution of pesticides in Heilongjiang Province[J]. Pesticide Science and Management, 2017(12): 9-10+14.

[2] Liao Xiaobin, Chen Meiqiu, Li Zhipeng, et al. Discussion on cultivated land quality protection incentive countermeasures based on the perspective of farmers' use of pesticides and fertilizers[J]. Zhejiang Agricultural Sciences, 2017(4): 673-676+678.

[3] Gu Xiaojun, Tian Sufen. Pesticides and cancer[J]. World Science and Technology Research and Development, 2005 (2): 47-52.

[4] $\mathrm{Hu} \mathrm{Xu,} \mathrm{Li} \mathrm{Lu,} \mathrm{Zhang} \mathrm{Qinfa,} \mathrm{etc.} \mathrm{Analysis} \mathrm{of} \mathrm{the} \mathrm{im-}$ pact of environmental hormone pollutants on food safety[J].Food Industry,2014 (9):230-234.

[5] Cheng Yan, Tan Lichao, Zhou Junying, et al. Screening of the priority list of environmental hormone pesticides in my country[J]. Pesticide Science and Management, 2017(4): 28-35.

[6] Run Wanming, Jiang Yiqun. Discussion on some problems of organophosphorus pesticides[J]. Inner Mongolia Petrochemical Industry, 2001(27): 52-54

[7] Yang Guang. Ministry of Agriculture and Rural Affairs: my country's pesticide utilization rate has reached $38.8 \%[\mathrm{~J}]$. Pesticide Market Information, 2018(11): 11-12.

[8] Teng Ruiju, Wang Xuemei, Wang Huan, et al. Research progress in the degradation and metabolism of organophosphorus pesticides[J]. Gansu Science and Technology, 2016(4): 46-50.

[9] Sun B, Zhang L X, Yang L Z, et al. Agricultural NonPoint Source Pollution in China: Causes and Mitigation Measures. AMBIO, 2012(4):370-379.

[10] Wang Shenghui, Zhang Chen, Yan Yanchun. Research progress in the microbial degradation of organophosphorus pesticides[J]. Biotechnology, 2006 (3): 95-97.

[11] Liang Yibo, Tan Xinghe, Hu Wangzi, etc. Research progress on degradation methods of pesticide residues in vegetables[J].Food Research and Develop- ment,2015(6):135-138.

[12] Yan Yanchun, Qiao Chuanling, Zhou Xiaotao. High enzyme activity of an engineered bacteria and degradation of pesticides by immobilized cells[J]. China Environmental Science, 2011(5): 461-465.

[13] Zhu He, Ji Mingshan. The latest development of pesticide residue rapid detection technology $[\mathrm{J}]$. Chinese Agricultural Science Bulletin, 2014(4): 242-250.

[14] Yu Hong, Chen An, Li Xiaojing, et al. Determination of 33 organophosphorus pesticide residues in vegetables by solid phase extraction combined with gas chromatography[J]. Chinese Journal of Food Hygiene, 2011(4): 330-334 .

[15] GB/T 5009.20-2003, Determination of Organophosphorus Pesticide Residues in Foods, issued on August 11, 2003, and implemented on January 1, 2004.

[16] Wang Wei, He Hongwu, Wang Lieping, et al. Organophosphorus pesticides and their research and development overview[J]. Pesticides, 2016(2): 86-90.

[17] You Minsheng, Liu Xin. Biodegradation and bioremediation of pesticide pollution[J]. Journal of Ecology, 2004(1): 73-77.

[18] Gu Yue, Jiang Hua. Microbial degradation of pesticide contaminated soil[J]. Liaoning Agricultural Sciences, 2013(4): 52-55.

[19] Liu Yuhuan, Zhong Yingchang. Study on Methamidophos Degrading Fungi[J]. China Environmental Science, 1999(2): 172-175.

[20] Wang Baihui, Jiao Hao, Liu Baolin, et al. Research progress in the mechanism of soil enzymes on pesticide degradation[J]. Chinese Agricultural Science Bulletin, 2012(32): 253-257.

[21] Zhao Renbang, Jin Cunhua, Liu Weihua. Immobilization of Penicillium oxalicum ZHJ6 on the degradation of methamidophos pesticides[J]. Chinese Agricultural Science Bulletin, 2012(26): 247-251.

[22] Gong Xiaojie, Li Ligong, Li Guanglei. Methyl parathion hydrolase mutant with improved enzymatic performance and its application [P], National Invention Patent (substantial examination), application number: 201710505744.2, publication number: 107189993A.

[23] Zhang Lijian, Zhu Lizhi, Wang Liping. Thoughts on developing "Chinese medicine agriculture" to promote sustainable agricultural development[J]. China Agricultural Information, 2016(11): 3-4.

[24] Zuo Changrui. New degrading enzymes effectively remove pesticide residues in vegetables[J]. Science and Technology Daily, 2011(8):53.

[25] Lin Lijing, Cheng Shenghua, Li Jihua, et al. The effect of tea tree oil on removing pesticide residues from cowpea[J]. Transactions of the Chinese Society of Agricultural Engineering, 2013(3): 273-278. 